\title{
Neue Beobachtungen zur Stereoselektivität von Monoiodcarben-Additionen
}

\author{
Eckehard V. Dehmlow*, Johanna Soufi, Hans-Georg Stammler ${ }^{l+1}$ und Beate Neumann $^{1 \neq 1}$ \\ Fakultät für Chemie, Universität Bielefeld, \\ Universitätsstraße 25, W-4800 Bielefeld 1
}

Eingegangen am 27. Juli 1992

Key Words: Iodocarbene, addition to alkenes / endo-Selectivity / Cyclooctadiene / Cyclooctatetraene

\section{New Observations Concerning the Stereoselectivity of Monoiodocarbene Additions}

Contrary to our earlier claim, $\mathrm{CHI}$ gives endo/exo isomer mixtures with cyclooctene. However, only endo-iodo compounds $7,8,10-12$ are obtained from COD and COT. Tricyclic compound 7 rearranges partially to bicyclic 9 . X-ray structure determinations of 7 and 9 were performed.
Die Chemie der Monoiodcarben-Addukte ist gegenüber der anderer Halogencarbene wegen der mangelnden $\mathrm{Me}$ thodenvielfalt zur Erzeugung von $\mathrm{CHI}$ und wegen der relativen Unbeständigkeit organischer Iodverbindungen unterentwickelt ${ }^{[1]}$. Vor kurzem wurde aus unserem Laboratorium u.a. über CHI-Additionen an cyclische Alkene mittlerer Ringgröße berichtet ${ }^{[2]}$. Die zunächst gefundene hohe endo-Selektivität veranlaßte die hier beschriebenen überprüfenden und weiterführenden Versuche.

Bei der photolytischen Erzeugung von CHI aus Iodoform in Gegenwart von $1 \mathbf{a}, \mathbf{1} \mathbf{b}$ oder $1 \mathbf{c}$ hatten wir ursprünglich ${ }^{[2]}$ 2a und 3a im Verhältnis 10:1 bzw. ausschließlich $\mathbf{2 b}$ und 2c gefunden. Wir haben die CHI-Versuche zunächst unter Variation der Bedingungen (Konzentrationen, Zugabemodus des Iodoforms, Wattleistung der Bestrahlungslampe u.a.) und Verfeinerung der Analytik (Gaschromatographie und 300-MHz-NMR-Spektroskopie) wiederholt. Es zeigt sich, daß auch $\mathbf{1} \mathbf{b}$ exo-Iodverbindung $3 \mathbf{b}^{[3]}$ liefert und daß<smiles>CCCCCC=CI</smiles>

1

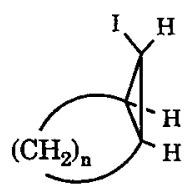

2

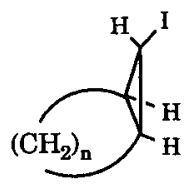

3

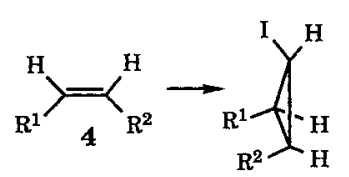

5

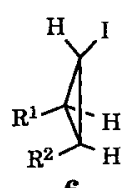

6
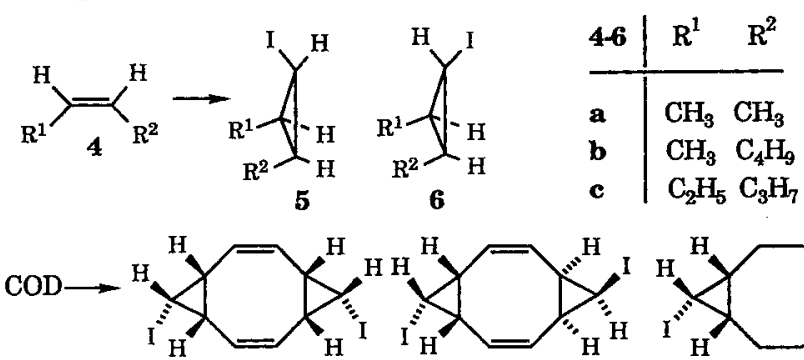

7

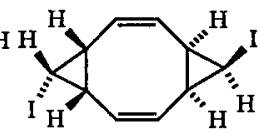

8

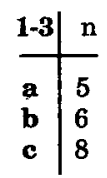

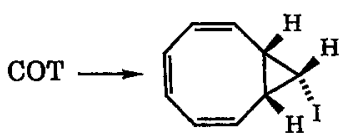

10

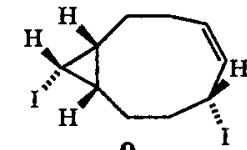

9

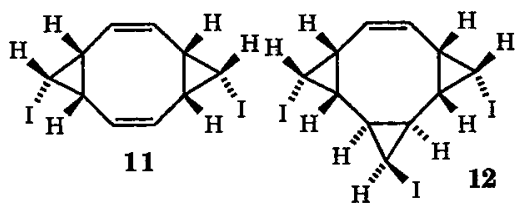

[₹] Röntgenstruktur-Analysen. bei Umsetzung von $1 \mathbf{a}$ und $\mathbf{1 b}$ eine relativ starke Variation des 2/3-Verhältnisses von Versuch zu Versuch auftritt, ohne $\mathrm{da} ß$ das auf einfache Weise mit den Reaktionsbedingungen zusammenhängt. Extremwerte waren: 2a/3a 8:2 und 6:4, 2b/3b 75:25 und 60:40. Bei 1c konnte kein zweites $C y$ cloaddukt aufgefunden werden. Es treten allerdings im 300MHz- ${ }^{1} \mathrm{H}-\mathrm{NMR}-$ Spektrum des rohen Reaktionsgemisches olefinische Signale auf, die von einem Zersetzungsprozeß von $2 c$ oder 3c herrühren könnten. Ein Iodalken konnte nicht rein erhalten werden. Wir erklären die Schwankungen mit der bekannten hohen Empfindlichkeit von Iodcyclopropanen (vgl. z. B. Lit. ${ }^{[7}$ ). Setzt man beim Aufarbeiten der Ansätze keine Base zu, so treten oftmals beim Einengen oder bei der Destillation plötzliche Polymerisationen oder sogar Verpuffungen auf. Andererseits ist bereits bekannt, daß zumindestens $\mathbf{2} \mathbf{b}$ und $\mathbf{3 b}$ unter den Reaktionsbedingungen und beim Rühren mit Kalium-tert-butylat in THF nicht verändert werden und da $B$ Monobromcarben mit 1c ebenfalls nur ein endo-Addukt gibt ${ }^{[2]}$.

Ausdehnung der Versuche auf die strukturähnlichen offenkettigen Verbindungen $4 \mathrm{~b}$ und $4 \mathrm{c}$ ergibt ebenfalls Isomerengemische, 5b/6b 65:35 bzw. 5c/6c 55:45. Für 5a/6a wurde das Verhältnis $6: 4$ schon früher bestimmt ${ }^{[2]}$.

Bei der CHI-Umsetzung von 1,5-Cyclooctadien wurde zuvor nur ein Bisaddukt (Schmp. 108-114 ${ }^{\circ} \mathrm{C}$ ) isoliert ${ }^{[2]}$, dem auf Grund des NMR-Spektrums und in Analogie zu literaturbekannten Fällen die endo-syn-endo-Stereochemie $7 \mathrm{zu}-$ gewiesen wurde. Mehrfache Säulenchromatographie liefert neben diesem Stoff (Schmp. $116^{\circ} \mathrm{C}$ ) zwei Isomere mit Schmp. 190 bzw. $60^{\circ} \mathrm{C}$. Die höher schmelzende Substanz hat im NMR-Spektrum sehr ähnliche Signallagen und Aufspaltungsmuster wie die Verbindung mit Schmp. $116^{\circ} \mathrm{C}$. Das Triplett für das jeweilige $\mathrm{H}$-Atom am Iod-tragenden $\mathrm{Cy}$ clopropan-C mit $J=7.6 \mathrm{bzw}$. $7.8 \mathrm{~Hz}$ zeigt, daß es sich eindeutig um 7 und 8 handeln muß. Die endgültige Zuordnung erfolgte durch eine Röntgenstrukturanalyse der Substanz mit Schmp. $116^{\circ} \mathrm{C}$ (vgl. Abb. 1). Sie erwies sich als endo-syn-endo-7, wie ursprünglich angenommen. Der Stoff mit Schmp. $190^{\circ} \mathrm{C} \mathrm{muB} \mathrm{demnach} \mathrm{endo-anti-endo-Isomer}$ 8 sein. Für das dritte Isomer wurde - u.a. zur Bestim- 
mung der relativen Stereochemie - ebenfalls eine Röntgenanalyse durchgeführt (vgl. Abb. 2). Es zeigte sich, daß $( \pm)-(1 \beta, 6 \alpha, 9 \beta, 10 \alpha)-6,10-$ Diodbicyclo[7.1.0]non-4-en (9) vorliegt.

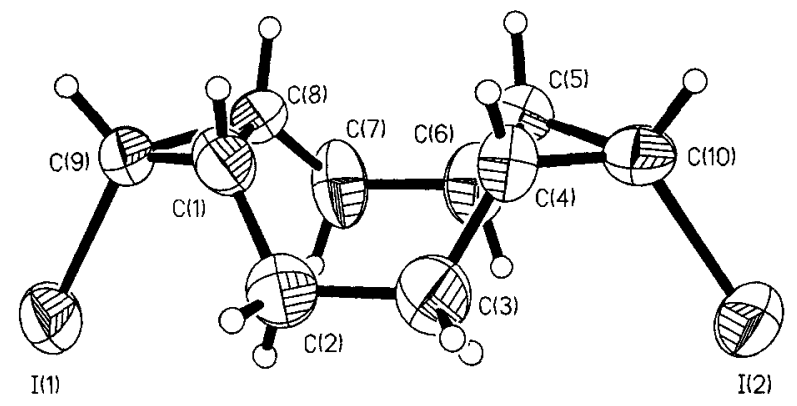

Abb. 1. Kristallstruktur von 7; ausgewählte Bindungslängen [pm] und -winkel [ ${ }^{\circ}$ ]: I(1) - C(9) 212.0(8), C(1) - C(9) 143.9(15), C(1) - C(8) 149.7(14), C(1)-C(2) 151.7(18); C(2)-C(1)-C(8) 122.6(10), C(1)$C(8)-C(9) \quad 56.9(6), C(1)-C(9)-C(8) \quad 60.6(7), C(8)-C(1)-C(9)$ $62.5(7), \mathrm{I}(1)-\mathrm{C}(9)-\mathrm{C}(1) 122.2(8), \mathrm{I}(1)-\mathrm{C}(9)-\mathrm{C}(8) 121.8(7)$

Offensichtlich ist diese Verbindung durch Isomerisierung eines primär gebildeten Biscarbenaddukts entstanden. Prinzipiell sind davon sechs Isomere möglich: endo-anti-endo (A $=8)$, endo-syn-endo $(\mathrm{B}=7)$, endo-anti-exo $(\mathrm{C})$, endo-synexo (D), exo-anti-exo (E) und exo-syn-exo (F). Nun sind in 9 beide lodatome $\alpha$-ständig, die Doppelbindung hat $Z$-Konfiguration, und der verbliebene Dreiring trägt sein Iodatom endo-ständig. Letzter Befund scheidet $\mathrm{E}$ und $\mathrm{F}$ aus. Bei der Umlagerung jeweils des einen Dreirings von A oder D bleiben die Iodatome auf unterschiedlichen Seiten ( $\alpha$ oder $\beta$ ) des Moleküls, so daß diese Isomere auch nicht in Frage kommen. Anwendung der Woodward-Hoffmann-De PuyRegeln $^{[4]}$ auf die verbleibenden Verbindungen $B$ und $C$ schließlich ergibt, daß nur $B=7$ ein $(Z)$-Alken liefern kann. Nach dieser Betrachtung muß also 9 aus 7 entstanden sein. Somit gibt es in diesem Falle keine Hinweise darauf, daß andere als endo-Iod-Isomere primär entstanden sind.

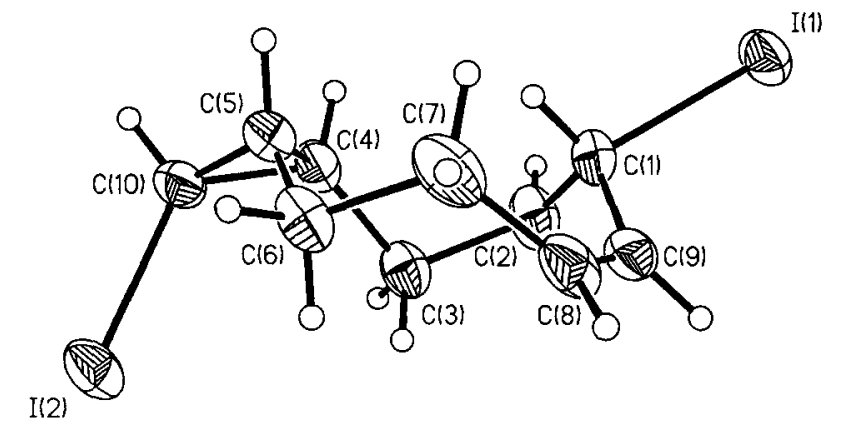

Abb. 2. Kristallstruktur von 9; ausgewählte Bindungslängen [pm] und -winkel [ ${ }^{\circ}$ ]: I(1) - C(1) 220.2(5), C(1)-C(9) 149.6(8), C(8)-C(9) $130.8(8), \quad C(5)-C(6) \quad 151.5(8), C(5)-C(10) 150.2(7), \quad I(2)-C(10)$ 211.4(5); I(1) - C(1)-C(2) 109.8(3), I(1)-C(1)-C(9) 108.7(3), $\mathrm{I}(2)-\mathrm{C}(10)-\mathrm{C}(4) 122.2(4), \mathrm{C}(5)-\mathrm{C}(4)-\mathrm{C}(10) 59.7(3), \mathrm{C}(4)-\mathrm{C}(5)-$ $C(10) 60.2(3), C(4)-C(10)-C(5) 60.1(3)$

Bindungsiängen und -winkel der Verbindungen 7 und 9 liegen im Rahmen des Normalen ${ }^{[5]}$ (vgl. auch Tab. 1, 2).
Cyclooctatetraen (COT) ist ebenfalls ein relativ starres Molekül. Man erhält bei der Umsetzung mit CHI das Monoaddukt 10 neben dem Bisaddukt 11. Die erste Verbindung fällt bei der Chromatographie im Gemisch mit COT und Iodoform an und konnte nur spektroskopisch charakterisiert werden, weil sie sehr labil ist. 10 und 11 sind auf Grund der Kopplungen im ${ }^{1} \mathrm{H}$-NMR wieder endo-Produkte. Bei 11 ist aus sterischen Gründen nur das endo-syn-endo-Isomer existenzfähig. Setzt man 11 erneut mit Iodcarben um, so erhält man in geringer Ausbeute das Triscarbenaddukt 12. Auch hier ist nur die gezeigte Stereochemie möglich. Die NMR-Spektren von 10-12 sind denen der bereits bekannten analogen Bromverbindungen sehr ähnlich ${ }^{[6]}$. Erneute längere Photolyse des reinen 11 liefert - wie zu erwarten - Naphthalin.

Insgesamt zeigt sich also, daß bestimmte Systeme in der Tat ausschließlich die vermutlich thermodynamisch weniger stabilen endo-Iodcarben-Addukte liefern. syn-bzw. endo-Selektivitäten großer Halogensubstituenten werden bei anderen Halogencarbenen mit einer anziehenden Wechselwirkung des hereinkommenden polarisierbaren Halogenrestes mit den benachbarten $\mathrm{C}-\mathrm{H}$-Bindungen qualitativ erklärt ${ }^{[1]}$. Wir beabsichtigen, die Selektivität an weiteren sterisch anspruchsvollen Alkenen, insbesondere Bicyclen, zu testen.

Diese Arbeit wurde durch den Fonds der Chemischen Industrie gefördert. E. V. D. dankt Prof. P. Jutzi für die Ermöglichung der Röntgenstrukturanalysen.

\section{Experimenteller Teil}

${ }^{1}$ H-NMR: Bruker AM 300, $\mathrm{CDCl}_{3}$ als Lösungsmittel und TMS als Standard. - Schmelzpunkte sind nicht korrigiert. - Siedepunkte der Kugelrohrdestillationen beziehen sich auf die Luftbadtemperatur. - GC: Carlo-Erba-Fractovap 4200, 3-m-OV 17-Säule. - Photolysen: Tauchlampe, Quecksilber-Hochdruckstrahler mit $\mathrm{CdI}_{2}$-Zusatz, TQ $718 \mathrm{Z3}$ der Fa. Heraeus mit bis zu $700 \mathrm{~W}$ Eingangsleistung.

Allgemeine Vorschrift für die Iodcarben-Additionen: Eine Lösung von $50 \mathrm{mmol}$ Alken in $600 \mathrm{ml}$ Dichlormethan sowie $31.5 \mathrm{~g} \mathrm{Na}_{2} \mathrm{SO}_{3}$ in $200 \mathrm{ml}$ Wasser werden in eine Photolyseapparatur mit der angegebenen Tauchlampe gebracht und bei $8-10^{\circ} \mathrm{C}$ intensiv gerührt. Innerhalb von $3 \mathrm{~h}$ werden $50 \mathrm{mmol}$ (im Falle von Dienen $100 \mathrm{mmol}$ ) fein zerstoßenes Iodoform in kleinen Anteilen zugegeben, wobei die Zugabe so reguliert wird, daß $\mathrm{CHI}_{3}$ immer bei Entfärbung nachdosiert wird. Anschließend wird noch 15 min weiterbestrahlt, dann aufgearbeitet: Die Phasen werden getrennt, die wäßrige wird mit wenig $\mathrm{CH}_{2} \mathrm{Cl}_{2}$ nachgewaschen und die vereinigte organische Lösung mit $\mathrm{Na}_{2} \mathrm{SO}_{4}$ getrocknet. Man setzt $500 \mathrm{mg}$ festes Natriummethanolat zu und entfernt unumgesetztes Alken und Dichlormethan i. Vak. Der Rückstand wird durch Chromatographie an Kieselgel oder durch Kugelrohrdestillation gereinigt und gegebenenfalls kristallisiert.

endo/exo-8-Iodbicyclo[5.1.0 Joctan (2a/3a): Nach der allgemeinen Vorschrift aus 1a; Sdp. 55-60 ${ }^{\circ} \mathrm{C} / 10$ Torr (Kugelrohr). 10-20\% Ausb. - Zusammensetzung laut GC und $300-\mathrm{MHz}^{-}{ }^{1} \mathrm{H}-\mathrm{NMR}$ zwischen $8: 2$ und 6:4. Charakteristisch sind für 2a $\delta 3.07$ (t, $J=$ $7.6 \mathrm{~Hz})$ und für $3 \mathrm{a} 2.12(\mathrm{t}, J=3.9)$.

$\mathrm{C}_{8} \mathrm{H}_{13} \mathrm{I}$ (236.1) Ber. C $40.70 \mathrm{H} 5.55$ Gef. C 40.65 H 5.41

endo/exo-9-Iodbicyclo/6.1.0Jnonan (2b/3b): Aus $1 \mathbf{b}$; $\mathrm{Sdp}$. $75-77^{\circ} \mathrm{C} / 0.7$ Torr (Kugelrohr). Ausb. bei verschiedenen Ansätzen 
9-53\%. - Zusammensetzung laut GC und $300 \mathrm{MHz}-\mathrm{NMR}$ zwischen 75:25 und 60:40. Charakteristisch sind für $2 \mathrm{~b} \delta 2.92(\mathrm{t}, J=$ $7.8 \mathrm{~Hz}$ ) und für $\mathbf{3 b} 1.83(\mathrm{t}, J=4.0)$.

\section{$\mathrm{C}_{9} \mathrm{H}_{15} \mathrm{I}$ (250.1) Ber. C 43.22 H 6.05 Gef. C 43.70 H 5.95}

endo-11-Iodbicyclo/8.1.0Jundecan (2c): Aus 1c; Sdp. $77^{\circ} \mathrm{C} / 0.05$ Torr (Kugelrohr). Ausb. 25\%. $-{ }^{1} \mathrm{H}-\mathrm{NMR}: \delta 2.93$ (t, $1 \mathrm{H}, J=7.8$ ), $2.45-0.8(\mathrm{~m}, 18 \mathrm{H}$ ). Minimale Absorption im Bereich $\delta$ 1.7-1.9 (wo das $\mathbf{t}$ für $3 \mathrm{c}$ läge).

\section{$\mathrm{C}_{11} \mathrm{H}_{19} \mathrm{I}(278.2) \quad$ Ber. C 47.50 H 6.88 Gef. C 47.32 H 6.67}

syn/anti-( $1 \alpha, 2 \alpha, 3 \alpha$ und 1 $\alpha, 2 \beta, 3 \beta)$-1-Butyl-2-iod-3-methylcyclopropan (5b/6b): Aus $4 \mathrm{~b}, \operatorname{Sdp}$. $55-60^{\circ} \mathrm{C} / 12$ Torr (Kugelrohr); $48 \%$ Ausb., Isomerenverhältnis 65:35 nach NMR. - ${ }^{1} \mathrm{H}-\mathrm{NMR}: \delta 2.93$ (t, $J=7.7 \mathrm{~Hz}$, von $5 \mathrm{~b}$ ), $1.81(\mathrm{t}, J=4.1$, von $6 \mathrm{~b}$ ), $1.5-0.7(\mathrm{~m})$.

\section{$\mathrm{C}_{8} \mathrm{H}_{15} \mathrm{I}$ (238.1) Ber. C 40.35 H 6.35 Gef. C 40.20 H 5.79}

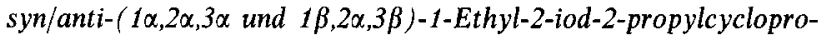
pan (5c/6c): Aus 4c, Sdp. $56-61^{\circ} \mathrm{C} / 11$ Torr (Kugelrohr); $20 \%$ Ausb., sehr zersetzliches Gemisch; Isomerenverhältnis 55:45 nach NMR. $-{ }^{1} \mathrm{H}$-NMR: $\delta 2.90(\mathrm{t}, J=7.7 \mathrm{~Hz}$, von $5 \mathrm{c}), 1.84(\mathrm{t}, J=4.2$, von $6 \mathrm{c}), 1.5-0.7(\mathrm{~m})$.

\section{$\mathrm{C}_{8} \mathrm{H}_{15} \mathrm{I}$ (238.1) Ber. C 40.35 H 6.35 Gef. C 40.52 H 6.01}

endo-syn-endo-( $1 \beta, 4 \beta, 5 \alpha, 6 \beta, 9 \beta, 10 \alpha)-5,10$-Diiodbicyclo[ $\left.7.1 .0 .0^{4,6}\right]$ decan (7): Aus COD. Bei der Kugelrohrdestillation des rohen Reaktionsgemisches geht zunächst bei $60-100^{\circ} \mathrm{C} / 12$ Torr eine Mischfraktion über, die im wesentlichen das bekannte 2-endo-Monoaddukt enthält. Die anschließende Ölpumpen-Vakuumdestillation bei ca. $80-130^{\circ} \mathrm{C} / 0.1$ Torr ergibt ein gelbbraunes Öl, das durch Mitteldruckchromatographie an Kieselgel mit Petrolether $\left(50-70^{\circ} \mathrm{C}\right)$ weiter aufgetrennt wird. Farblose Kristalle, Schmp. $116^{\circ} \mathrm{C}, 5 \%$ Ausb. $-{ }^{1} \mathrm{H}-\mathrm{NMR}$ : $\delta 3.09(\mathrm{t}, 2 \mathrm{H}, J=7.8 \mathrm{~Hz}), 2.02(\mathrm{~m}$, $4 \mathrm{H}), 1.43(\mathrm{~m}, 4 \mathrm{H}), 1.00$ (sehr breites s, $4 \mathrm{H}$ ).

\section{$\mathrm{C}_{10} \mathrm{H}_{14} \mathrm{I}_{2}$ (388.0) Ber. C 30.95 H 3.64 Gef. C 31.20 H 3.80}

endo-anti-endo- $(1 \alpha, 4 \beta, 5 \alpha, 6 \beta, 9 \alpha, 10 \beta)-5,10$-Diiodbicyclo[ $\left.7.1 .0 .0^{4.6}\right]$ decan (8), als spätere Fraktion der o. a. Chromatographie, farblose Kristalle, Schmp. $190^{\circ} \mathrm{C}, 1.7 \%$ Ausb. $-{ }^{1} \mathrm{H}-\mathrm{NMR}: \delta 2.94(\mathrm{t}, 2 \mathrm{H}$, $J=7.6), 2.04(\mathrm{~m}, 4 \mathrm{H}), 1.29(\mathrm{~m}, 4 \mathrm{H}), 0.91$ (sehr breites s, $4 \mathrm{H})$.

\section{$\mathrm{C}_{10} \mathrm{H}_{14} \mathrm{I}_{2}$ (388.0) Ber. C 30.95 H 3.64 Gef. C 31.01 H 3.78}

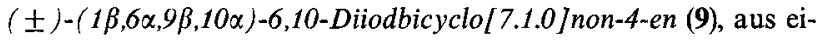
ner weiteren Fraktion der o.a. Chromatographie, erst nach Wochen kristallin erhalten, Schmp. $60^{\circ} \mathrm{C}, 1.5 \%$ Ausb. - ${ }^{1} \mathrm{H}-\mathrm{NMR}$ : $\delta 5.75-5.5(\mathrm{~m}, 2 \mathrm{H}), 5.35-5.2(\mathrm{~m}, 1 \mathrm{H}), 2.84(\mathrm{t}, 1 \mathrm{H}, J=7.8 \mathrm{~Hz})$, $2.45-1.95(\mathrm{~m}, 6 \mathrm{H}), 1.15-0.95(\mathrm{~m}, 3 \mathrm{H}), 0.8-0.75(\mathrm{~m}, 1 \mathrm{H})$.

\section{$\mathrm{C}_{10} \mathrm{H}_{14} \mathrm{I}_{2}$ (388.0) Ber. C 30.95 H 3.64 Gef. C 31.20 H 3.64}

endo-9-Iodbicyclo[6.1.0]nona-2,4,6-trien (10): Aus COT. Bei der Kugelrohrdestillation (Sdp. $40^{\circ} \mathrm{C} / 0.1$ Torr) wurde eine zersetzliche Fraktion erhalten $(\approx 5 \%)$, die nicht zur Analyse gebracht werden konnte. $-{ }^{1} \mathrm{H}$-NMR: $\delta 6.06$ (verbreitertes d, $2 \mathrm{H}, J=11.7 \mathrm{~Hz}$ ), 5.85 (verbreitertes s, $2 \mathrm{H}$ ), 5.66 (d mit weiterer kleiner Aufspaltung, $2 \mathrm{H}$ ), 3.10 (t, $1 \mathrm{H}, J=7.9$ ), 1.82 (d, $2 \mathrm{H}, J=7.9$ ).

endo,syn,endo- $(1 \beta, 4 \beta, 5 \alpha, 6 \beta, 9 \beta, 10 \alpha)-5,10$-Diiodbicyclo [7.1.0.0 $\left.0^{4,6}\right]$ deca-2,7-dien (11): Durch Chromatographie des Destillationsrückstandes an Kieselgel mit Petrolether $\left(50-70^{\circ} \mathrm{C}\right)$ und Kristallisation; $4 \%$ Reinausb., Schmp. $160-163^{\circ} \mathrm{C}$ (Zers.). - ${ }^{1}$ H-NMR: $\delta 5.33$ (s, $4 \mathrm{H}), 3.17(\mathrm{t}, 2 \mathrm{H}, J=7.6 \mathrm{~Hz}), 2.07(\mathrm{~d}, 4 \mathrm{H})$.

\section{$\mathrm{C}_{10} \mathrm{H}_{10} \mathrm{I}_{2}$ (384.0) Ber. C 31.27 H 2.65 Gef. C $31.60 \mathrm{H} 2.94$}

$(1 \beta, 4 \beta, 5 \alpha, 6 \beta, 7 \alpha, 8 \beta, 9 \alpha, 10 \beta, 11 \alpha)-5,8,11$-Triiodtetracyclo $\left[8.1 .0 .0^{4.6}\right.$ $\left.0^{1,10}\right]$ undec-2-en (12): Aus 11 durch erneute Umsetzung nach der allgemeinen Vorschrift; anschließend chromatographisch abgetrennt und kristallisiert. Zers.-P. ab $150^{\circ} \mathrm{C}$, ca. $2 \%$ Reinausb. -
${ }^{1} \mathrm{H}-\mathrm{NMR}: \delta 5.54(\mathrm{~s}, 2 \mathrm{H}), 3.33(\mathrm{t}, 2 \mathrm{H}, J=7.7 \mathrm{~Hz}), 3.31(\mathrm{t}, 1 \mathrm{H}, J=$ $8.0), 1.79(\mathrm{t}, 2 \mathrm{H}, J=8.0), 1.3-1.2(\mathrm{~m}, 2 \mathrm{H}), 0.9-0.8(\mathrm{~m}, 2 \mathrm{H})$.

$\mathrm{C}_{11} \mathrm{H}_{11} \mathrm{I}_{3}$ (523.9) Ber. C $25.22 \mathrm{H} 2.12$ Gef. C 25.23 H 2.17

Röntgenstrukturanalysen: Mit Vierkreisdiffraktometer Siemens P2 ${ }_{1}$; Strahlung Mo- $K_{\alpha} ; \lambda=71.073$ pm; Graphitmonochromator; Meßmethode: $\omega$-scan; Programm: SHELXTL-PLUS (VMS); Strukturlösung: Direkte Methoden; Full-matrix-Verfeinerung; alle NichtWasserstoffatome anisotrop; alle Wasserstoffatome [außer an $\mathrm{C}(8)$ und C(9) bei 9] an berechneten Positionen mit fixem $U_{\text {eq }}=80 \mathrm{pm}^{2}$ $\times 10^{-1}$.

7: Formel $\mathrm{C}_{10} \mathrm{H}_{14} \mathrm{I}_{2}$, Molmasse $388.032 ;$ Kristallgröße $0.15 \times 0.3$ $\times 0.5 \mathrm{~mm}$; Gitterkonstanten $a=1087.9(3), b=1077.3(3), c=$ $1072.9(2) \mathrm{pm}, \beta=113.95(2)^{\circ} ; V=1149.0(4) \cdot 10^{6} \mathrm{pm}^{3} ;$ K ristallsystem monoklin; Raumgruppe $P 2_{1} / c ; Z=4 ; d_{\text {ber }}=2.248 \mathrm{~g} / \mathrm{cm}_{3} ; \mu=$ $5.382 \mathrm{~mm}^{-1} ; F(000) 720 ; T=293 \mathrm{~K}$; Meßbereich $3^{\circ} \leqslant 2 \Theta \leqslant 60^{\circ}$; Zahl der gemessenen Reflexe $3704( \pm h ;-k ;-l)$, davon 3365 unabhängige mit $F \geqslant 4 \sigma(F)$; Anzahl der verfeinerten Parameter 109; Reflex-Parameter-Verhältnis $15.5: 1 ; R=0.057 ; R_{w}=0.048 ; w^{-1}$ $=\sigma^{2}\left(F_{\mathrm{o}}\right)$; max. Restelektronendichte $1.24 \cdot 10^{-6} \mathrm{e} / \mathrm{pm}^{3}$ and $\mathrm{I}$.

9: Formel, Molmasse wie 7; Kristallgröße $0.15 \times 0.3 \times 0.3 \mathrm{~mm}$; Gitterkonstanten $a=755.4(5), b=886.2(2), c=1748.9(9) \mathrm{pm}$, $\beta=93.03(5)^{\circ} ; V=1169.1(11) \cdot 10^{6} \mathrm{pm}^{3} ;$ Kristallsystem monoklin; Raumgruppe $P 2_{\mathfrak{t}} / c ; Z=4 ; d_{\text {ber }}=2.205 \mathrm{~g} / \mathrm{cm}^{3} ; \mu=5.277 \mathrm{~mm}^{-1}$; $F(000) 720 ; T=173 \mathrm{~K}$; Meßbereich $3^{\circ} \leqslant 2 \Theta \leqslant 55^{\circ}$; Zahl der gemessenen Reflexe $3092(+h ;+k ; \pm h$, davon 2704 unabhängige mit $F \geqslant 5 \sigma(F)$; Positionen der H-Atome an $\mathrm{C}(8)$ und $\mathrm{C}(9)$ mit fixem $U_{\text {eq }}=80 \mathrm{pm}^{2} \cdot 10^{-1}$ verfeinert; Anzahl der verfeinerten Parameter 115; Reflex-Parameter-Verhältnis 17.4:1; $R=0.031 ; R_{w}=0.028$; $w^{-1}=\sigma^{2}\left(F_{0}\right) ;$ max. Restelektronendichte $0.63 \times 10^{-6} \mathrm{e} / \mathrm{pm}^{3}$.

Tab. 1. Atomkoordinaten $\left(\cdot 10^{4}\right)$ und äquivalente isotrope Auslenkungsparameter $\left[\mathrm{pm}^{2} \cdot 10^{-1}\right]$ für 7. Äquivalente isotrope $U$ berechnet als ein Drittel der Spur des orthogonalen $U_{i j}$-Tensors

\begin{tabular}{llcll}
\hline & $\mathbf{x}$ & $\mathrm{y}$ & $\mathrm{z}$ & $\mathrm{U}(\mathrm{eq})$ \\
\hline $\mathrm{I}(1)$ & $6844(1)$ & $-904(1)$ & $9303(1)$ & $68(1)$ \\
$\mathrm{I}(2)$ & $-598(1)$ & $-1318(1)$ & $7571(1)$ & $72(1)$ \\
$\mathrm{C}(1)$ & $4333(10)$ & $-2221(10)$ & $7101(10)$ & $49(5)$ \\
$\mathrm{C}(2)$ & $3932(10)$ & $-2697(12)$ & $8213(10)$ & $68(6)$ \\
$C(3)$ & $2450(10)$ & $-2757(11)$ & $7910(10)$ & $60(5)$ \\
$\mathrm{C}(4)$ & $1487(9)$ & $-2481(11)$ & $6481(10)$ & $50(5)$ \\
$\mathrm{C}(5)$ & $1280(10)$ & $-1155(9)$ & $5964(10)$ & $47(4)$ \\
$\mathrm{C}(6)$ & $2007(10)$ & $-104(11)$ & $6862(11)$ & $62(6)$ \\
$\mathrm{C}(7)$ & $3517(10)$ & $12(10)$ & $7205(12)$ & $66(6)$ \\
$\mathrm{C}(8)$ & $4109(9)$ & $-905(9)$ & $6612(9)$ & $43(4)$ \\
$C(9)$ & $5502(9)$ & $-1475(10)$ & $7326(9)$ & $47(4)$ \\
$C(10)$ & $192(11)$ & $-1840(10)$ & $6130(10)$ & $53(5)$ \\
\hline
\end{tabular}

Tab. 2. Atomkoordinaten $\left(\cdot 10^{4}\right)$ und äquivalente isotrope Auslenkungsparameter $\left[\mathrm{pm}^{2} \cdot 10^{-1}\right]$ für 9. Äquivalente isotrope $U$ berechnet als ein Drittel der Spur des orthogonalen $U_{i j}$-Tensors

\begin{tabular}{lrrrr}
\hline & \multicolumn{1}{c}{$\mathrm{x}$} & $\mathrm{y}$ & \multicolumn{1}{c}{$\mathrm{z}$} & $\mathrm{U}(\mathrm{eq})$ \\
\hline $\mathrm{I}(1)$ & $12571(1)$ & $1266(1)$ & $93(1)$ & $41(1)$ \\
$\mathrm{I}(2)$ & $6196(1)$ & $2264(1)$ & $3440(1)$ & $42(1)$ \\
$\mathrm{C}(1)$ & $10689(6)$ & $1972(6)$ & $944(3)$ & $30(2)$ \\
$\mathrm{C}(2)$ & $11307(7)$ & $1395(7)$ & $1733(3)$ & $39(2)$ \\
$\mathrm{C}(3)$ & $9849(6)$ & $1667(7)$ & $2312(3)$ & $33(2)$ \\
$\mathrm{C}(4)$ & $8199(6)$ & $808(6)$ & $2062(3)$ & $30(2)$ \\
$\mathrm{C}(5)$ & $6583(7)$ & $1525(6)$ & $1669(3)$ & $33(2)$ \\
$\mathrm{C}(6)$ & $6378(7)$ & $3190(6)$ & $1490(3)$ & $34(2)$ \\
$\mathrm{C}(7)$ & $7156(7)$ & $3651(7)$ & $714(3)$ & $42(2)$ \\
$\mathrm{C}(8)$ & $8959(9)$ & $4351(7)$ & $816(3)$ & $38(2)$ \\
$\mathrm{C}(9)$ & $10480(8)$ & $3649(6)$ & $903(3)$ & $34(2)$ \\
$\mathrm{C}(10)$ & $6482(7)$ & $896(6)$ & $2463(3)$ & $36(2)$ \\
\hline
\end{tabular}


[1] E. V. Dehmlow in: Methoden der Organischen Chemie (HoubenWeyl), Bd. E9b/Tl. 2 (Hrsg.: M. Regitz), G. Thieme Verlag, Stuttgart, 1989, S. $1461-1627$.

${ }^{[2]}$ E. V. Dehmlow, J. Stütten, Tetrahedron Lett. 1991, 43, 6105.

${ }^{[3]}$ Das Isomer 3b ist in der Dissertation J. Stütten, Universität Bielefeld, 1991 (vgl. Lit. ${ }^{[2]}$ ), ganz offensichtlich wegen Verwendung nur von $60-\mathrm{MHz}-{ }^{1} \mathrm{H}-\mathrm{NMR}$-Spektren und Verzicht auf geeignete GC-Säulen übersehen worden.

${ }^{[4]}$ C. H. DePuy, Acc. Chem. Res. 1968, 1, 33.
${ }^{[5]}$ Weitere Einzelheiten zur Kristallstrukturuntersuchung können beim Fachinformationszentrum Karlsruhe, Gesellschaft für wissenschaftlich-technische Information mbH, D-7514 EggensteinLeopoldshafen 2, unter Angabe der Hinterlegungsnummer CSD-56631, der Autoren und des Zeitschriftenzitats angefordert werden.

${ }^{[6]}$ E. V. Dehmlow, M. Lissel, Liebigs Ann. Chem. 1979, 181.

${ }^{[7]}$ R. Mathias, P. Weyerstahl, Angew. Chem. 1974, 86, 42; Angew. Chem. Int. Ed. Engl. 1974, 13, 132

$[296 / 92]$ 